\title{
Maladies à transmission vectorielle, changements climatiques et modes de vie sains en milieu urbain : prochaines étapes
}

\author{
Mathieu $\mathrm{K}^{1}$, Karmali $\mathrm{M}^{1 *}$ \\ Affiliation \\ ${ }^{1}$ Institut des politiques sociales et de la santé de l'Université McGill, Montréal (Québec) \\ *Correspondance : mkarmali@rogers.com
}

Citation proposée : Mathieu K, Karmali M. Maladies à transmission vectorielle, changements climatiques et modes de vie sains en milieu urbain : prochaines étapes. Relevé des maladies transmissibles au Canada 2016;42:243-5. https://doi.org/10.14745/ccdr.v42i10a13f

\section{Introduction}

Les résumés dans le présent numéro traitent des principales présentations données au cours d'un atelier tenu à Montréal en avril 2016 (1) afin de discuter de maladies à transmission vectorielle (MTV) dans les villes canadiennes d'un point de vue interdisciplinaire, dans le contexte des changements climatiques et des modes de vie sains en milieu urbain (2-11). Un problème clé examiné était: Quels sont les liens entre les changements climatiques et les modes de vie sains et qu'est-ce qu'ils signifient pour l'avenir des maladies à transmission vectorielle dans les villes canadiennes? Le résumé suivant vise à mettre en évidence les points clés de l'atelier, leur interconnectivité, ainsi que les besoins, les lacunes et les occasions qui se dégagent des présentations et des discussions.

\section{Le contexte mondial}

Les MTV, telles que la dengue, l'infection par le virus Chikungunya, et l'infection par le virus Zika, qui sont habituellement transmises par certaines espèces du moustique Aedes, apparaissent ou étendent les menaces liées aux maladies infectieuses à l'échelle mondiale, en particulier dans les pays à revenu faible et intermédiaire. Les facteurs qui peuvent les amener à se manifester comprennent l'urbanisation rapide incontrôlée et la croissance de la population, le surpeuplement, le manque de logements adéquats et d'infrastructures sanitaires, les changements climatiques, l'évolution des agents pathogènes, et l'aggravation de la lutte contre les vecteurs (1-4). Dans les régions endémiques de l'Afrique et de l'Asie, la dengue, le virus Chikungunya et le virus Zika étaient généralement maintenus par des hôtes du réservoir faunique et plusieurs espèces de moustiques. Les effets indirects sur les populations humaines sont survenus lorsque les gens étaient infectés par contact avec des moustiques dans les contrées sauvages. De nos jours, ces maladies sont maintenues presque entièrement dans des cycles de transmission interhumaine. Par conséquent, les maladies transmises par les moustiques se propagent dans de nouvelles régions du monde où il existe des vecteurs compétents, sans que les animaux prennent part à des cycles de transmission, ce qui augmente le risque d'éclosion à l'échelle mondiale. Cette tendance est accentuée davantage en raison d'un réchauffement climatique, ce qui peut favoriser la réplication du vecteur et la transmission de la maladie dans des régions non endémiques auparavant (3). En outre, l'émergence et l'expansion des MTV dans les pays aux ressources limitées ayant une capacité de surveillance et des systèmes de santé faibles amplifie le risque de propagation à l'échelle mondiale (3). Ensemble, ces nouvelles tendances mettent l'accent sur la nécessité d'une coordination et de partenariats de façon à éliminer les problèmes émergents que posent les MTV dans le monde et au Canada (3).

\section{Les maladies à transmission vectorielle (MTV) au Canada}

Dans le passé, les MTV préoccupantes pour la santé publique étaient rares au Canada, mais au cours des dernières années, I'infection par le virus du Nil occidental transmise par les moustiques et la maladie de Lyme transmise par les tiques ont émergé comme d'importantes menaces pour la santé publique $(4,5)$. L'infection par le virus du Nil occidental est désormais la maladie transmise par les moustiques la plus courante au Canada. Ce virus est conservé dans la nature par l'entremise de cycles entre les moustiques du genre Culex et certaines espèces d'oiseaux. La maladie de Lyme, causée par la bactérie Borrelia burgdorferi, est la maladie transmise par les tiques la plus courante au Canada. Cette tique infectée est maintenue chez les petits mammifères tels que les souris. À l'heure actuelle, les vecteurs des deux maladies se sont adaptés aux milieux urbains, et ils vivent, se nourrissent et se reproduisent à proximité des humains. Le virus du Nil occidental est désormais endémique au Canada, bien que son incidence varie d'une année à l'autre. L'incidence de la maladie de Lyme augmente constamment à mesure que la zone géographique couverte par les tiques s'étend (4-6).

Le Canada possède des programmes de surveillance qui assurent efficacement le suivi de la maladie de Lyme et l'infection par le virus du Nil occidental (5). Des inquiétudes subsistent concernant le risque de progression des MTV « exotiques » telles que l'infection par les virus Chikungunya et Zika vers le nord par rapport à nos voisins du sud, en raison de l'augmentation des températures à l'échelle mondiale, qui favorise la réplication des moustiques Aedes et la transmission du virus $(4,5)$. 


\section{Répercussions du milieu urbain et des changements climatiques sur les maladies à transmission vectorielle (MTV)}

De plus en plus de données probantes indiquent que le milieu urbain bâti, y compris l'étalement urbain, constitue un facteur de risque pour les maladies chroniques comme l'obésité, le diabète et les problèmes respiratoires. Ces maladies découlent en partie d'une dépendance à l'égard des voitures pour le transport et d'une diminution des occasions de pratiquer une activité physique (6-8). La réduction de l'étalement urbain, les meilleures connexions entre les villes et la transformation de celles-ci afin qu'elles soient plus propices à la marche, avec des parcs et des espaces verts et bleus, peuvent contribuer à un mode de vie plus sain (6-8).

L'étalement urbain a également un certain nombre d'effets écologiques en raison de son effet sur la couverture terrestre et le microclimat local, l'eau, la flore et la faune, ainsi que la disponibilité de l'habitat pour les espèces vectrices et hôtes et la capacité des vecteurs. La croissance, la survie et le comportement des moustiques et des tiques sont extrêmement sensibles aux températures environnementales qui, à leur tour, sont influencées par les effets d'îlot de chaleur du milieu urbain. Cela signifie que les températures dans les zones urbaines sont plus élevées que dans les régions rurales environnantes (6). Les efforts visant à atténuer les effets d'îlot de chaleur en créant plus d'endroits verts et bleus, comme des parcs et des lacs, pourraient favoriser la réplication des moustiques, en particulier s'ils sont combinés au réchauffement climatique (8). Ces efforts peuvent aussi être aggravés par les piscines d'eau stagnante lorsqu'ils sont associés à certains types d'aménagement paysager et de conception de réseaux de drainage, aussi bien dans les zones urbaines que dans les zones suburbaines $(3,4,6,8)$.

\section{Mesures d'atténuation et d'adaptation}

L'augmentation des températures à l'échelle mondiale constitue un facteur de risque important en matière de propagation des MTV émergentes dans les pays aux climats tempérés. Bien que l'atténuation des changements climatiques soit devenue une priorité à l'échelle mondiale, elle demeure un défi permanent avec peu de possibilités d'amélioration à court et moyen terme. Parmi les stratégies, on vise la prise en charge des déterminants sociaux de la santé (en particulier dans les pays à revenu faible ou intermédiaire), la réduction des effets d'îlot de chaleur et de l'étalement urbain, et la promotion de principes d'aménagement rehaussant un mode de vie sain tout en réduisant au minimum les possibilités de reproduction des vecteurs.

Il convient de classer par ordre de priorité la surveillance accrue des MTV qui sont actuellement endémiques, notamment la maladie de Lyme et le virus du Nil occidental (9), le suivi étroit des MTV (9) possibles arrivant dans des régions exposées à un risque d'émergence, ainsi que la détection rapide des cas pour l'arrivée de MTV exotiques liée à des voyages $(4,5)$. II est fortement recommandé de développer une stratégie de communication à l'intention du public concernant la réduction du risque ainsi qu'une stratégie pour les médecins concernant le diagnostic et le traitement (9). Les messages au sujet des mesures de protection personnelle doivent également être adaptées afin de tenir compte de l'évolution des populations urbaines et de la réaction de la génération $Y$ face aux risques et aux menaces en milieu urbain (10).

Enfin, il existe un besoin urgent d'évaluer et de perfectionner davantage les méthodes de contrôle des vecteurs. Par exemple, on utilise des insecticides pyréthroïdes, larvicides et d'autres produits chimiques, mais les données relatives à leur efficacité et leur innocuité sont limitées (11).

\section{Besoins, lacunes et possibilités}

Il y a un consensus général sur la nécessité de continuer à militer fortement contre les changements climatiques afin de réduire le risque de MTV. Cette mobilisation pourrait comprendre les activités suivantes :

- Améliorer la collaboration interdisciplinaire et multisectorielle (8);

- Surmonter la mentalité de cloisonnement qui a tendance à se développer au sein et à l'échelle des secteurs de politique et de pratique (8);

- Informer les personnes travaillant dans le domaine de la santé et de la politique publiques et des affaires urbaines au sujet des nouvelles connaissances et recommandations;

- Élargir les recherches aux fins suivantes :

- Élaborer des modèles de prédictions grâce à des outils tels que le système d'information géographique (SIG) en vue d'améliorer l'évaluation des risques d'apparition de MTV associés à la migration, aux voyages à l'étranger, ainsi qu'aux modèles de peuplement $(2,4)$;

- Étudier les risques et les avantages des insecticides, y compris les pyréthroïdes et les larvicides (11);

- Recueillir davantage de données sur les comportements de groupes de population précis, particulièrement en ce qui a trait à la prise de risques, aux voyages à l'étranger et à l'évolution des habitudes et des intérêts, qui augmentent le risque de transmission des MTV (10);

- Étudier les éléments moteurs de l'émergence et la transmission des MTV afin de concevoir de meilleurs programmes d'intervention (3);

- Étudier les interactions entre les déterminants climatiques et sociaux afin de mieux comprendre leur lien complexe avec l'apparition potentielle de MTV dans les villes (2);

- Analyser les coûts et les répercussions économiques des interventions par rapport au fait de ne prendre aucune mesure.

Les lacunes au niveau local pourraient être comblées comme suit :

- Comprendre les tendances en matière de comportement qui stimulent l'étalement urbain et la dynamique de la population à l'intérieur et autour des centres urbains, afin d'aider à élaborer des politiques efficaces à tous les niveaux (6); 
- Comprendre les types particuliers d'aménagement paysager ( $p$. ex. les zones isolées par rapport aux régions naturelles reliées) qui peuvent accroître ou réduire le taux de reproduction, la survie et l'alimentation des vecteurs, surtout dans les environnements bâtis, et optimiser la planification urbaine et les stratégies d'utilisation des terres dans les zones urbaines et périurbaines, afin de minimiser les risques de MTV (6);

- Intervenir à l'échelle locale pour répondre aux changements climatiques, car leurs effets sont ressentis à l'échelle locale;

- Améliorer le régime de réglementation pour contrôler l'étalement urbain $(6,8)$.

Voici les stratégies à l'échelle internationale :

- Mettre en œuvre les objectifs stratégiques de développement des Nations Unies en vue de régler le problème des MTV et d'autres maladies infectieuses émergentes (1);

- Prendre des mesures continues contre les changements climatiques.

En ce qui concerne les prochaines étapes, on a proposé un symposium structuré; dans le cadre de celui-ci, les invités prépareront des prises de position détaillées et un ordre du jour sera exprimé pour la recherche approfondie, ce qui pourrait mener à des demandes ciblées en matière de recherche interdisciplinaire concertée (8).

\section{Conclusion}

À la fois dans un contexte mondial et au Canada, les MTV sont les plus préoccupantes lorsqu'elles se trouvent le contexte des changements climatiques et des modifications dans les modes d'utilisation des terres. L'environnement bâti des villes est un déterminant important de la santé. Un aménagement urbain avisé peut contribuer à faire baisser les taux de maladies chroniques et à limiter les répercussions des MTV. Cet atelier, comptant des participants provenant des différents secteurs et disciplines, a réussi à promouvoir une reconnaissance des nombreux différents points de vue qui doivent être pris en compte afin d'examiner l'avenir dans un contexte de changement climatique et de coordonner les efforts pour un partenariat lié à la santé mondiale.

\section{Conflit d'intérêts}

Aucun

\section{Références}

1. Alabaster GP. Enjeux de politique mondiaux liés aux risques de maladies à transmission vectorielle. Relevé des maladies transmissibles au Canada 2016;42:227-8. http://www.phac- aspc.gc.ca/publicat/ccdr-rmtc/16vol42/dr-rm42-10/ar-05-fra. php.

2. Berrang-Ford L, Harper SL, Eckhardt R. Maladies à transmission vectorielle : mettre fin au débat entre déterminants climatiques et déterminants sociaux. Relevé des maladies transmissibles au Canada 2016;42:235-6. http://www.phac-aspc.gc.ca/publicat/ccdr-rmtc/16vol42/drrm42-10/ar-09-fra.php.

3. Kulkarni MA. Propagation et répercussions des maladies à transmission vectorielle émergentes à l'échelle mondiale. Relevé des maladies transmissibles au Canada 2016;42;2212. http://www.phac-aspc.gc.ca/publicat/ccdr-rmtc/16vol42/ dr-rm42-10/ar-02-fra.php.

4. Ogden NH. Maladies à transmission vectorielle, changement climatique et conception urbaine. Relevé des maladies transmissibles au Canada 2016;42:225-6. http://www.phacaspc.gc.ca/publicat/ccdr-rmtc/16vol42/dr-rm42-10/ar-04-fra. php.

5. Lindsay LR. Situation actuelle des maladies à transmission vectorielle communes au Canada. Relevé des maladies transmissibles au Canada 2016;42:223-4. http://www.phacaspc.gc.ca/publicat/ccdr-rmtc/16vol42/dr-rm42-10/ar-03-fra. php.

6. Jaeger JAG, Nazarnia N. Répercussions sociales et écologiques de la croissance exponentielle de l'étalement urbain à Montréal. Relevé des maladies transmissibles au Canada 2016;42:231-2. http://www.phac-aspc.gc.ca/ publicat/ccdr-rmtc/16vol42/dr-rm42-10/ar-07-fra.php.

7. Booth GL. Environnement bâti et santé. Relevé des maladies transmissibles au Canada 2016;42:229-30. http://www.phacaspc.gc.ca/publicat/ccdr-rmtc/16vol42/dr-rm42-10/ar-06-fra. php.

8. Luka N. Nouveaux enjeux des maladies à transmission vectorielle pour les villes canadiennes. Relevé des maladies transmissibles au Canada 2016;42:241-2. http://www.phacaspc.gc.ca/publicat/ccdr-rmtc/16vol42/dr-rm42-10/ar-12-fra. php.

9. Lowe AM. Stratégies d'intervention en santé publique visant à contrer les maladies à transmission vectorielle au Québec. Relevé des maladies transmissibles au Canada 2016;42:2378. http://www.phac-aspc.gc.ca/publicat/ccdr-rmtc/16vol42/ dr-rm42-10/ar-10-fra.php.

10. Boudreau JA. Comprendre les réactions au risque. Relevé des maladies transmissibles au Canada 2016;42:233-4. http://www.phac-aspc.gc.ca/publicat/ccdr-rmtc/16vol42/drrm42-10/ar-08-fra.php.

11. Chevrier J. Équilibrer les risques et les bienfaits de I'utilisation des insecticides pour le contrôle des vecteurs : I'étude Venda Health Examination of Mothers, Babies and their Environment (VHEMBE). Relevé des maladies transmissibles au Canada 2016;42:239-40. http://www.phacaspc.gc.ca/publicat/ccdr-rmtc/16vol42/dr-rm42-10/ar-11-fra. php. 